\title{
EFECTO DEL ESTADIO DEL DESARROLLO FOLICULAR AL MOMENTO DE LA MONTA SOBRE LA OVULACIÓN Y SUPERVIVENCIA EMBRIONARIA EN ALPACAS
}

\author{
Effect of the Follicular Development Stage at the Time of Mating on the \\ Ovulation and Embryonic Survival in Alpacas
}

Miriam Cervantes F. ${ }^{1}$, Wilfredo Huanca L. ${ }^{1,3}$ y Teodosio Huanca M. ${ }^{2}$

\section{RESUMEN}

El objetivo del presente estudio fue evaluar el efecto del estadio del desarrollo folicular del folículo dominante (fd) al momento de la cópula, sobre la ovulación y supervivencia embrionaria en alpacas. Se utilizaron 116 animales con descanso post-parto $\geq 15$ días, que fueron evaluadas por ecografía transrectal para distribuirlas en 4 grupos: G1 (fd en estadio de crecimiento, diámetro: $6 \mathrm{~mm}$ ), G2 (fd en estadio de crecimiento, diámetro: $\geq 7 \mathrm{y} \leq 12$ $\mathrm{mm}$ ), G3 (fd en estadio estático, diámetro: $\geq 7 \mathrm{~mm}$ ) y G4 (fd en estadio de regresión, diámetro: $\geq 7 \mathrm{~mm}$ ). Posteriormente, fueron sometidos a monta controlada, a excepción de 5 alpacas del grupo G1 que rechazaron al macho. El día del empadre fue considerado el día 0 . Evaluaciones ecográficas adicionales se realizaron los días 2 (ocurrencia de ovulación), 9 (presencia y tamaño del cuerpo lúteo); 20, 25, 30 y 35 (presencia de vesícula embrionaria o embrión). El día 15 post cópula se realizó la prueba de receptividad sexual. El 97.3\% de alpacas empadradas $(n=111)$ presentaron ovulación al día 2 post cópula, sin diferencia significativa $(\mathrm{p}<0.05)$ entre grupos. En el día 9 post cópula, no se encontraron diferencias significativas en el tamaño promedio del cuerpo lúteo entre grupos. El porcentaje de supervivencia embrionaria fue estadísticamente similar para todos los grupos, aunque hubo una tendencia a un mayor nivel de supervivencia para el grupo G2 (65.5\%) en comparación con los demás grupos al día 35 post cópula. Estos resultados indicarían que el estadio del desarrollo folicular del folículo dominante al momento de la monta no tendría efecto significativo sobre la tasa de ovulación y supervivencia embrionaria en alpacas.

Palabras clave: alpaca, ecografía, desarrollo folicular, ovulación, supervivencia embrionaria

${ }^{1}$ Laboratorio de Reproducción Animal, Facultad de Medicina Veterinaria, Universidad Nacional Mayor de San Marcos, Lima

${ }^{2}$ Estación Experimental ILLPA, Instituto Nacional de Investigacion Agropecuaria, Puno

${ }^{3}$ E-mail: whuanca@appaperu.org 


\section{Abstract}

The objective of this study was to evaluate the effect of the stage of the dominant follicle (df) at mating on the ovulation and embryonic survival in alpacas. A total of 116 alpacas with $\geq 15$ day-post-partum resting period were used and evaluated by transrectal ultrasonography in order to distribute them in 4 groups: G1 (df in growing stage, diameter: $6 \mathrm{~mm}$ ), G2 (df in growing stage, diameter: $\geq 7 \leq 12 \mathrm{~mm}$ ), G3 (df in static stage, diameter: $\geq 7 \mathrm{~mm}$,) and G4 (df in regression stage, diameter: $\geq 7 \mathrm{~mm}$ ). Subsequently, all alpacas were mated except 5 alpacas of group G1 that rejected the male. Mating day was considered as day 0. Additional ultrasound evaluations were carried out on days 2 (occurrence of ovulation), 9 (presence and size of the corpus luteum); 20, 25, 30 and 35 (presence of embryonic vesicle orembryo). On day 15, a sexual receptiveness test was performed. Ovu lation occurred in 97.3\% of alpacas that were mated $(n=111)$ but without significant differences $(p<0.05)$ between groups. On day 9 , no significant difference in the average size of the corpus luteum was observed among groups. The embryonic survival rate was also not significantly different among the groups, but there was a tendency to a greater rate of survival for group $G 2(65.5 \%)$ on day 35 aftermating. These results suggested that the stage of follicular development of the dominant follicle during mating does not have a significant effect on the rate of ovllation and embryonic survival in alpacas.

Key words: alpaca, ultrasonography, follicular development, ovulation, embryonic survival

\section{INTRODUCCIÓN}

Una de las principales limitantes de desarrollo en las explotaciones alpaqueras es la baja eficiencia reproductiva, principalmente por efecto de la alta mortalidad embrionaria (Novoa, 1991). Uno de sus posibles factores podría estar relacionado con el estadio de desarrollo del folículo dominante al momento de la monta, considerando que la alpaca puede aceptar al macho en cualquier estadio (Bravo et al., 1991; Sumar, 1997) y que puede permanecer en estado de receptividad constante hasta por 40 días, debido a la presencia de un folículo estrogénico que se desarrolla en ondas foliculares continuas de larga duración (Novoa, 1989; Fernández Baca, 1993).

La ovulación ocurre 26 a 30 horas después de la cópula (San Martín et al., 1968; Adams et al., 1990), habiendo fallas ovulatorias post cópula de origen desconocido, atribuidas a una sensibilidad disminuida de los folículos a los niveles circulantes de
LH en determinados estadios de maduración folicular (Fernández Baca, 1971; Novoa, 1989). Se reconoce que alpacas con folículos pequeños liberan cantidades menores de LH y que no ocurre ovulación en aquellas con folículos $<7 \mathrm{~mm}$ de diámetro al momento de la cópula (Bravo et al., 1991), lo que estaría relacionado con la adquisición de los receptores de LH en estados avanzados del desarrollo folicular (Gore-Langton y Armstrong, 1994).

Los índices de fertilización a los tres días post servicio son superiores al $85 \%$. Los porcentajes de alpacas que mantienen la gestación a los 30 días post servicio son mucho menores, estimándose una pérdida embrionaria del 50\% (Fernández Baca et al., 1970a; Fernández Baca, 1971).

La progesterona del cuerpo lúteo (CL) es necesaria para el mantenimiento de la preñez en la alpaca (Novoa, 1991; Sumar, 1997); por lo menos hasta el décimo mes (Novoa, 1991), de allí que se requiere su adecuado crecimiento después de la ovulación. Se co- 
noce que la ovulación es difícil que ocurra en folículos en regresión (Bravo et al., 1991; Hafez, 1996), y de ocurrir la ovulación, el CL resultante puede ser insuficiente ocurriendo una mortalidad embrionaria temprana (Sumar, 1997). Por tanto, el objetivo del presente trabajo fue evaluar el efecto de los estadios de crecimiento, estático y regresión del folículo dominante al momento de la cópula, sobre la ovulación y la supervivencia embrionaria en alpacas.

\section{Materiales y Métodos}

\section{Lugar de estudio}

El experimento se realizó en el Anexo Quimsachata de la Estación Experimental ILLPA-INIA, ubicada a $4200 \mathrm{msnm}$, entre los distritos de Santa Lucía y Cabanillas de las provincias de Lampa y San Román, respectivamente, en el departamento de Puno. La zona está a $15^{\circ} 04^{\prime}$ de latitud sur y a $70^{\circ} 18^{\prime}$ de longitud oeste. El estudio se realizó durante los meses de febrero y marzo del 2003.

\section{Animales}

Se usaron 116 alpacas hembras adultas con historia de partos anteriores y con descanso postparto $\geq 15$ días y con presencia de folículos $(>5 \mathrm{~mm}$ ) detectados por ecografía transrectal ovárica usando un ecógrafo modelo ALOKA SSD 500 y un transductor lineal de 7.5 MHz. Todas las hembras seleccionadas recibieron las mismas condiciones de manejo y fueron alimentadas en pastura natural.

\section{Diseño experimental}

Luego del descanso postparto, se realizaron evaluaciones ecográficas diarias por tres días consecutivos para determinar el estadio de desarrollo folicular (crecimiento, maduración o regresión). En base a estas mediciones, los animales fueron asignados a uno de los siguientes grupos:
G1 ( $n=27)$ : folículo dominante en estadio de crecimiento (diámetro $=6 \mathrm{~mm}$ ).

G2 ( $n=30)$ : folículo dominante en estadio de crecimiento (diámetro $\geq 7 \mathrm{y} \leq 12$ $\mathrm{mm})$.

G3 ( $n=30)$ : folículo dominante en estadio estático (diámetro $\geq 7 \mathrm{~mm}$ )

G4 (n=29): folículo dominante en estadio de regresión (diámetro $\geq 7 \mathrm{~mm}$ ).

El tamaño del folículo dominante durante los tres días de observación aumentó (G1 y G2), mantuvo (G3), o redujo (G4) su tamaño, siendo el tamaño del $3^{\text {er }}$ día el correspondiente a su grupo.

Una vez asignadas a uno de los tratamientos, las alpacas fueron sometidas a monta controlada con machos del plantel de reproductores.

\section{Procedimiento metodológico}

El día de la monta fue considerado el día 0 y evaluaciones ecográficas adicionales se realizaron en los días 2, 9, 20, 25, 30 y 35 . El día 2 se confirmó la ocurrencia de ovulación en base al criterio de desaparición del folículo dominante; en el día 9 se determinó la presencia y tamaño del cuerpo lúteo y en los días 20, 25, 30, 35 se determinó la presencia de la vesícula embrionaria o embrión. Adicionalmente, en el día 15 post cópula se realizó la prueba de receptividad sexual, enfrentando a las hembras con un macho; clasificándolas como receptivas y posibles vacías a aquellas que adoptaron la posición de cópula, y como no receptivas y posibles preñadas a las que no aceptaron al macho.

\section{Análisis estadístico}

Los datos fueron analizados utilizando el paquete estadístico STATA 8.0 (Statistical Analysis, 2003). La prueba de análisis de varianza (ANOVA) de una sola vía fue utilizada para analizar la diferencia entre grupos con respecto al tamaño del cuerpo lúteo al día 9 post cópula. La relación entre la tasa de ovulación con los grupos en estudio fueron evaluadas con la prueba de Chi Cuadrado. 
Cuadro 1. Porcentaje de ovulación en alpacas con folículo en fase de crecimiento (G1 y G2), fase estática (G3) y fase de regresión (G4) al momento de la monta

\begin{tabular}{ccccc}
\hline \multirow{2}{*}{ Grupos } & \multicolumn{2}{c}{$\mathrm{N}^{\mathrm{o}}$ de alpacas } & \multicolumn{2}{c}{ Ovulación } \\
\cline { 2 - 5 } & Sometidas a monta & Aceptan la monta & $\mathrm{n}$ & $\%$ \\
\hline G1 & 27 & 22 & 21 & 95.5 \\
G2 & 30 & 30 & 29 & 96.7 \\
G3 & 30 & 30 & 30 & 100.0 \\
G4 & 29 & 29 & 28 & 96.6 \\
\hline Total & 116 & 111 & 108 & 97.3 \\
\hline
\end{tabular}

G1: crecimiento (Folículo dominante $=6 \mathrm{~mm}$ ); G2: crecimiento ( $\geq 7 \mathrm{y} \leq 12 \mathrm{~mm})$; G3: estático $(\geq 7 \mathrm{~mm})$; G4: regresión $(\geq 7 \mathrm{~mm})$

Cuadro 2. Frecuencia (\%) de supervivencia embrionaria ${ }^{1}$ en alpacas con folículo en fase de crecimiento (G1 y G2), fase estática (G3) y fase de regresión (G4) al momento de la monta

\begin{tabular}{cccccc}
\hline Día post cópula & G1 & G2 & G3 & G4 & Total \\
\hline $15^{2}$ & 66.7 & 72.4 & 60.0 & 50.0 & 62.3 \\
20 & 57.1 & 69.0 & 60.0 & 50.0 & 59.0 \\
25 & 57.1 & 65.5 & 60.0 & 42.9 & 56.4 \\
30 & 57.1 & 65.5 & 56.7 & 42.9 & 55.6 \\
35 & 52.4 & 65.5 & 53.3 & 42.9 & 53.5 \\
\hline
\end{tabular}

G1: crecimiento (Foliculo dominante $=6 \mathrm{~mm}$ ); G2: crecimiento ( $\geq 7 \mathrm{y} \leq 12 \mathrm{~mm}$ ); 3: estático $(\geq 7 \mathrm{~mm})$; G4: regresión $(\geq 7 \mathrm{~mm})$

${ }^{1}$ Estimado por ecografia

${ }^{2}$ Estimado por receptividad sexual

La supervivencia embrionaria en los diferentes días de observación y final fue analizada mediante curvas de supervivencia de Kaplan Meier. Para la comparación de supervivencia final entre grupos se utilizó la prueba de Log-Rank. El nivel de significancia empleado para el análisis de los resultados fue de $\mathrm{p}<0.05$.

\section{Resultados}

\section{Tasa de ovulación}

E1 $97.3 \%$ de las alpacas que fueron servidas $(\mathrm{n}=111)$ presentaron ovulación al día 2 post cópula y formación de cuerpo lúteo en 


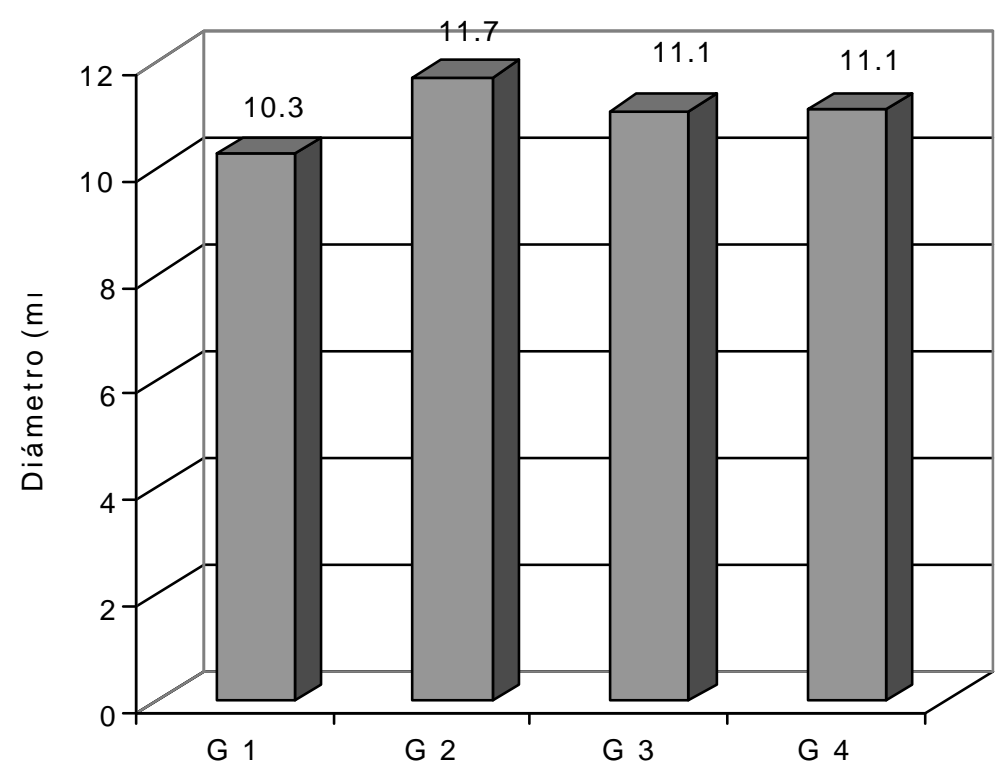

Figura 1. Tamaño promedio del cuerpo lúteo en el día 9 post cópula en alpacas confolículo en fase de crecimiento ( $\mathrm{G} 1: 6 \mathrm{~mm}, \mathrm{G} 2: \geq 7 \mathrm{y} \leq 12 \mathrm{~mm}$ ), fase estática (G3: $\geq 7 \mathrm{~mm}$ ), y fase de regresión (G4: $\geq 7 \mathrm{~mm}$ ) al momento de la monta

el día 9, sin diferencias significativas entre los grupos en estudio (Cuadro 1). Cinco alpacas del grupo G1 no fueron sexualmente receptivas al macho por lo que no fueron servidas.

\section{Tasa de supervivencia embrionaria}

La tasa de supervivencia embrionaria experimentó una progresiva disminución que continuó hasta el último día de observación. No se encontró diferencia significativa entre los grupos en estudio (Cuadro 2), aún cuando al día 35 post cópula se registra una tendencia a un mayor nivel de supervivencia para el grupo G2.

\section{Tamaño del cuerpo lúteo el día 9 post cópula}

No se encontraron diferencias significativas en el tamaño promedio del cuerpo lúteo entre los grupos en estudio en el día 9 post cópula (Fig. 1).

\section{Discusión}

Los resultados del presente estudio sugieren que las tasas de ovulación y supervivencia embrionaria no estarían afectadas por el estadio del desarrollo folicular al momento de la monta.

La receptividad sexual de la hembra en el día de la monta en G1, a excepción de los otros grupos, fue de $81.5 \%$. Se sabe que la alpaca acepta al macho en cualquier estadio folicular (Bravo et al., 1991; Sumar, 1997); sin embargo, el rechazo al macho por las alpacas del grupo G1 podría explicarse por la escasa habilidad de los folículos pequeños de secretar cantidades significativas de estrógenos (Gore-Langton y Armstrong, 1994).

Las alpacas del grupo G2, quienes tenían folículo dominante promedio de $8.7 \pm 1.5$ $\mathrm{mm}$ en fase de crecimiento al momento de la cópula, tuvieron una relativa mayor frecuen- 
cia de supervivencia embrionaria $(65 \%)$ al día 35 post cópula comparados con los demás grupos en estudio. Si se considera que la duración total del estadio de crecimiento es de $4.8 \pm 1.5$ días (Bravo et al., 1990), que varios de estos folículos se hallarían en la etapa final de la fase de crecimiento y que la ovulación ocurre a las 26 horas post cópula (San Martín et al., 1968), se podría concluir que la ovulación de estos folículos ocurrió en el estadio estático, momento de mejor respuesta para el establecimiento y mantenimiento del cuerpo lúteo (Sumar, 1997).

En base a lo anterior, también se podría explicar la razón de no haber encontrado el mejor porcentaje de supervivencia embrionaria en el grupo G3 (estático). Los folículos dominantes de este grupo $(8.8 \pm 1.4$ $\mathrm{mm}$ ), tomando en cuenta la duración total de estadio de maduración ( $5.0 \pm 1.6$ días; Bravo et al., 1990), se encontrarían en un estadio avanzado de maduración, con relativa proximidad al estadio de regresión, en el momento de la ovulación.

Los resultados del grupo G4 (regresión) indicaron la ocurrencia de ovulación seguida de fertilización, contrastando con otros trabajos (Bravo et al., 1991) que señalan que ocurre luteinización, mas no ovulación por efecto de la monta. Sin embargo, dependiendo del grado de alteración de la calidad del folículo dominante, a pesar de no afectarse su capacidad ovulatoria, se vería afectada la supervivencia embrionaria (Mihm et al., 1999); lo que concordaría con los resultados del presente estudio, donde este grupo tuvo el menor porcentaje de supervivencia embrionaria $(42.9 \%)$.

El rechazo al macho por algunas alpacas del G1 podría deberse a la escasa habilidad de los folículos pequeños de secretar cantidades significativas de estrógenos (GoreLangton y Armstrong, 1994). Por otro lado, la ocurrencia de ovulación en alpacas del grupo G1, que presentaban foliculos pequeños, estaría explicado por los cambios en la es- tructura folicular del folículo dominante y por la actividad proliferativa que ocurre en el intervalo entre el pico de LH y la ovulación (Gore-Langton y Armstrong, 1994); adquiriendo la capacidad ovulatoria por el incremento de la expresión de receptores de LH en las células de la granulosa (Sartori et al., 2001).

El tamaño promedio del cuerpo lúteo para todos los grupos en estudio, fue similar y no presentó diferencia significativa al día 9 post cópula, día en el cual alcanzaría el máximo desarrollo (Fernández Baca et al., 1970b). Estos resultados sugieren que tras la monta, la formación del cuerpo lúteo sería independiente del estadio del folículo del cual proviene, aunque probablemente, su mantenimiento sí podría estar influenciado por el estadio folicular pre ovulatorio.

\section{Conclusiones}

- La tasa de ovulación fue superior al 95\%, independientemente del estadio de desarrollo folicular al momento de la monta en alpacas.

- El estadio del desarrollo folicular no tendría efecto significativo sobre la tasa de supervivencia embrionaria en alpacas.

- Alpacas con folículo dominante en estadio de crecimiento $\geq 7 \mathrm{~mm}$ al momento de la monta, presentaron la mayor tasa de supervivencia embrionaria $(65.5 \%)$ y las alpacas con folículo dominante en estadio de regresión tuvieron la supervivencia embrionaria más baja (42.9\%).

\section{Literatura Citada}

1. Adams G, Sumar J, Ginther O. 1990. Effects of lactational and reproductive status on ovarian follicular waves in llamas (Lama glama). J Reprod Fertil 90: 535-545. 
2. Bravo PW, Fowler M, Stabenfeldt G, Lasley B. 1990. Ovarian follicular dynamics in the llama. Biol Reprod 43: 579-585.

3. Bravo PW, Stabenfeldt G, Lasley B, Fowler M. 1991. The effect of ovarian follicle size on pituitary and ovarian responses to copulation in domesticated South American Camelids. Biol Reprod 45: 553-559.

4. Fernández Baca S. 1971. La Alpaca. Reproducción y crianza. Boletín $\mathrm{N}^{\circ} 7$. IVITA-UNMSM. Lima. 43 p.

5. Fernández Baca S. 1993. Manipulation of reproductive functions inmale and female New World camelids. Anim Reprod Sci 33: 307-323.

6. Fernández Baca S, Hansel W, Novoa C. 1970a. Embryonic mortality in the alpaca. Biol Reprod 3: 243-251.

7. Fernández Baca S, Hansel W, Novoa C. 1970b. Corpus luteum function in the alpaca. Biol Reprod 3: 252-261.

8. Gore-Langton R, Armstrong D. 1994. Follicular steroidogenesis and its control. En: Knobil \& Nelly (eds). The physiology of reproduction. $2^{\text {nd }}$ ed. New York: Raven Press. p 571-628.
9. Mihm M, Curran N, Hyttel P, Knight P, Boly M, Roche J. 1999. Effect of dominant follicle persistence on follicular fluid oestradiol and inhibin and on oocyte maturation in heifers. J Reprod Fertil 116: 293-304.

10. Novoa C. 1989. Reproducción. Simposio de producción de alpacas y llamas. XII Reunión Científica Anual APPA. p 67-72.

11. Novoa C. 1991. Fisiología de la reproducción de la hembra. En: Fernández Baca, S. (ed). Avances y perspectivas del conocimiento de los camélidos sudamericanos. Santiago de Chile: FAO. p 93-103.

12. San Martín M, Copaira M, Zúñiga J, Rodríguez R, Bustinza G, Acosta L. 1968. Aspects of reproduction in the alpaca. J Reprod Fertil 16: 395-399.

13. Sartori R, Fricke P, Ferreira F, Ginther O, Wiltbank M. 2001. Follicular deviation and adquisition of ovulatory capacity in bovine follicles. Biol Reprod 65: 1403-1409.

14. Sumar J. 1997. Avances y perspectivas en reproducción de camélidos. Memorias del I Symposium Internacional sobre Avances en Reproducción de Rumiantes. Lima, Perú. p 30-44. 